\title{
COMMUNICATION
}

Cite this: Lab Chip, 2013, 13, 4011

Received 24th June 2013,

Accepted 28th July 2013

DOI: $10.1039 / c 31$ c50755f

www.rsc.org/loc

\section{A novel method for sample delivery and testing of whole blood: gel strip PCR for point of care (POC) molecular diagnostics $\dagger$}

\author{
Dammika P. Manage, ${ }^{a}$ Jana Lauzon, ${ }^{a}$ Alexey Atrazhev, ${ }^{a}$ Xiaoli Pang ${ }^{\text {bc }}$ and Linda \\ M. Pilarskiªb
}

Testing of whole blood in miniaturized PCR is compromised by the opaque nature of whole blood that leads to physical masking of a fluorescent signal. We demonstrate a method to perform real-time PCR with whole blood that avoids interference from the opacity of whole blood.

Hydrogel cassette PCR contains all reagents needed for PCR in a desiccated form. ${ }^{1}$ Here, the cassette PCR was modified to accept whole blood. PCR reagents were polymerized into semi-solid gel strips $(\sim 13 \mu \mathrm{L})$ that are attached to a glass coverslip. The cassette PCR is assembled such that blood is introduced under the desiccated gel. Thus, fluorescent images can be acquired from the topside of the gel without any masking by whole blood clots that form during PCR cycling and are confined to the underside of each gel strip. A cassette can contain 12 strips in the current design and each strip can detect a different sample, with no cross contamination. This procedure allows using the unpurified blood for PCR without the need for cumbersome DNA extraction protocols. The use of gel strips can readily be adapted to a manufacturable chip that is self-contained and cold chain transportable for any POC application using whole blood.

POC testing for molecular diagnosis replaces centralized laboratory testing and provides efficient and fast diagnosis at lower cost, in a near-POC setting. This enables more informed medical decision-making to improve patient outcomes in a timely manner. An important application is to monitor for blood-borne infections, for example to identify pathogens causing unknown fevers (e.g. malaria, hepatitis) ${ }^{2-5}$ or BK viremia which occurs in immunocompromised transplant patients. ${ }^{6}$ If implemented on an automated POC device, molecular diagnostics could be readily used and results rapidly obtained near the bedside for monitoring

${ }^{a}$ Department of Oncology, University of Alberta and Cross Cancer Institute, 11560 University Avenue, Edmonton, AB, T6G 1Z2, Canada.E-mail: lpilarsk@ualberta.ca; Fax: +1780432 8425; Tel: +17804328925

${ }^{b}$ Department of Laboratory Medicine and Pathology, University of Alberta, $8440-112$ Street, Edmonton, AB, T6G 2B7, Canada

${ }^{c}$ Provincial Laboratory for Public Health, 8440 - 112 St, Edmonton, AB, T6G 2J2, Canada

† Electronic supplementary information (ESI) available. See DOI: 10.1039/c3lc50755f or triage. POC testing could be performed by non-laboratory staff without any need for complex sample preparation steps that would otherwise be difficult to implement. This goal for PCR testing requires direct testing of unprocessed sample and a diagnostic readout that is not compromised by opaque components in raw samples, particularly those in blood.

In our previous paper, ${ }^{1}$ we showed that the DNA amplification (PCR) can be performed in a gel capillary cassette that is selfcontained and storable. We showed the testing of up to 16 patients in one cassette for up to 4 sexually transmitted diseases with raw genital swabs and raw urine. The gels were desiccated inside glass capillaries to store the cassette for long term storage. When the gel is desiccated a path is created between the dried gel and the capillary walls for the sample to be delivered by capillary force. These samples were relatively clear, with no particulate material that would otherwise obscure the gel. However, if the sample contains opaque cellular materials that cannot enter the gel, as is the case with whole blood, these remain between the gel and the glass capillary walls. When heat is applied during PCR cycling, these substances create a barrier of solid mesh acting to mask laser light that would otherwise enter the gel and excite the intercalating dyes in real time PCR. The clotted blood mesh also masks the fluorescence signal (emission) from real time PCR, compromising measurement of PCR amplification. Because of this, PCR amplification cannot be detected due to the masking by blood components. In order to avoid masking of light when testing blood, we developed a novel technique that involves gel strips instead of gel capillaries. The gel in gel strips is attached to a coverslip to enable formation of a channel under the desiccated gel strip. Whole blood can be delivered to the underside of the gel strip. Clots that form during cycling are physically separated from the "face-up" regions of the hydrogel cassette where fluorescence excitation of intercalating dye occurs; these excited dye emissions are measured for real time PCR and amplicon melt curve analysis (MCA). As previously described for gel capillaries, ${ }^{1}$ gel strips are enclosed in wax which makes up the structure of a cassette and acts to prevent cross contamination between gel strip reaction units enclosing different primer sets and/or loaded with different samples. 
(a)

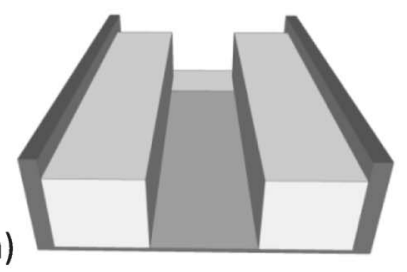

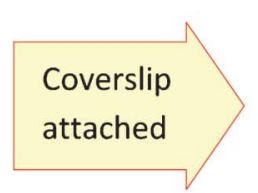

(b)

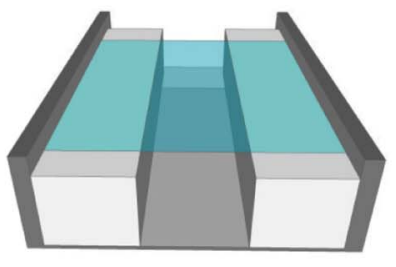

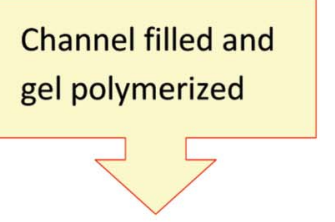

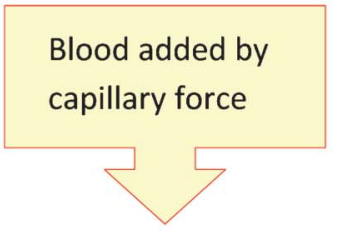

(d)
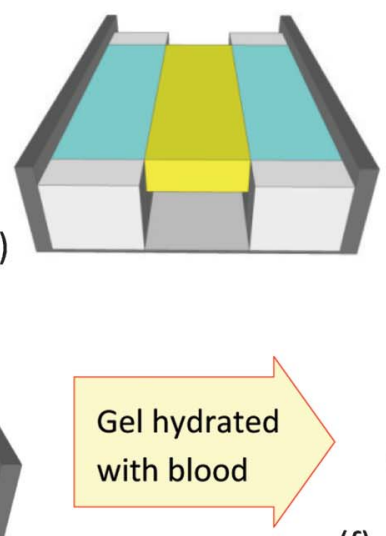

(e)

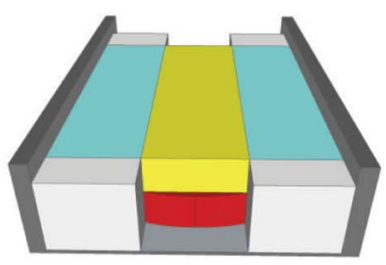

(c)

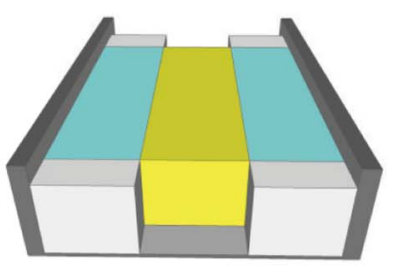

(f)

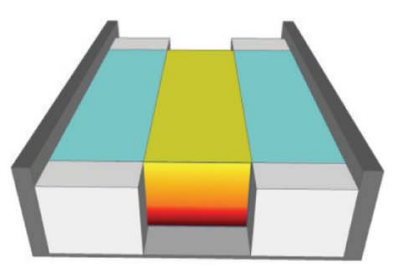

Fig. 1 Principle of gel strip cassette for PCR amplification from whole blood: (a) wax trenches are patterned in the pan with a PDMS seal, (b) a glass coverslip is

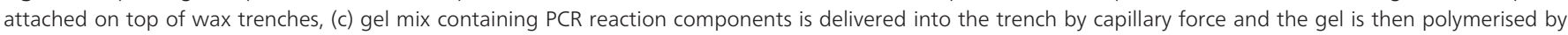

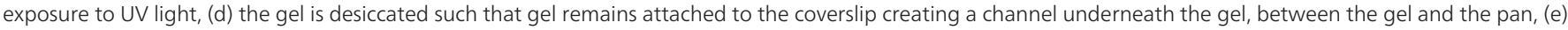

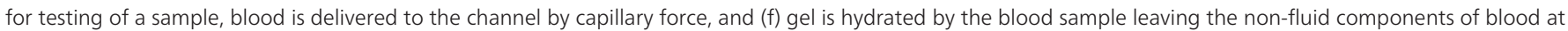
the bottom of the pan.

We have previously published method to perform whole blood PCR in liquid media. ${ }^{7}$ Malaria has also been detected in unprocessed blood by conventional PCR. ${ }^{8}$ Here we used a custom-constructed DNA polymerase that is resistant to inhibition by whole blood. Our goal was to analyse unprocessed blood in a pre-assembled and self-contained chip. As proof of principle for detecting pathogen templates in whole blood using the gel strip method, BK Virus (BKV) DNA was spiked into whole blood. BK viremia is an important cause of graft loss in kidney transplant recipients. $^{6}$
The gel strip assembly is made on the laboratory bench with "off the shelf" materials. A step by step procedure for making gel strips is shown in Fig. 1. A wax imprint was made on an aluminium pan $(23.5 \mathrm{~mm} \times 32 \mathrm{~mm})$ using a PDMS seal to create trenches with $2 \mathrm{~mm}$ width and $1 \mathrm{~mm}$ depth (Fig. 1(a)) with $750 \mu \mathrm{L}$ of wax (Surgipath Paraplast X-tra, Leica Microsystems). A $5 \mathrm{~mm}$ wide treated coverslip for adhering the gel (treated as described previously ${ }^{9}$ ) was attached on top of the wax trenches by applying heat, thereby creating channels for making the gel strips. Each channel can hold $13.5 \mu \mathrm{L}$ of reaction mix. The primers for amplifying a $100 \mathrm{bp}$ product in the BK virus were reported

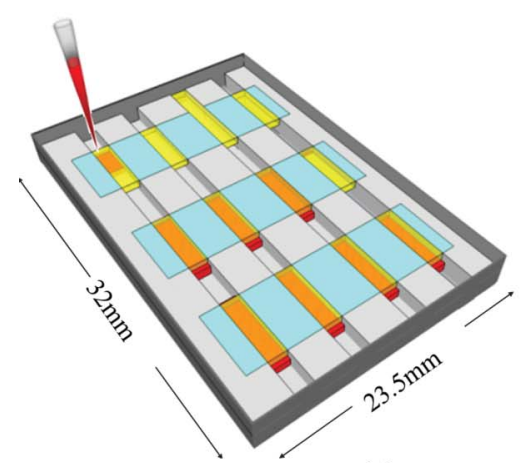

(a)

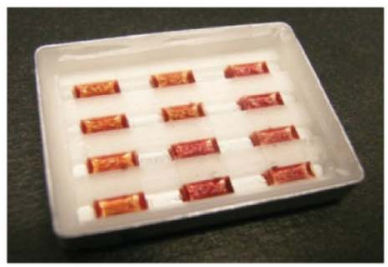

(b) (i)

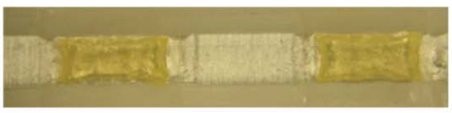

(ii)

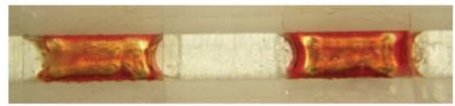

(iii)

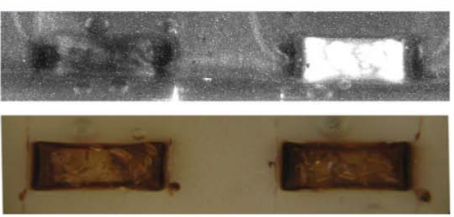

(c)

Fig. 2 Delivering blood to gel strip cassettes. (a) Cartoon of a gel strip cassette to test 12 blood samples (b) photograph of a cassette after the blood is delivered (c) photographs of gel strips -top view (i) after the desiccation and (ii) after the blood sample (8.5 $\mu \mathrm{L}$ per strip) is added, (iii) a CCD image acquired after the PCR amplification and (iv) a photograph of the strips after the PCR -left strip -negative for BKV and right strip -positive for BKV. 

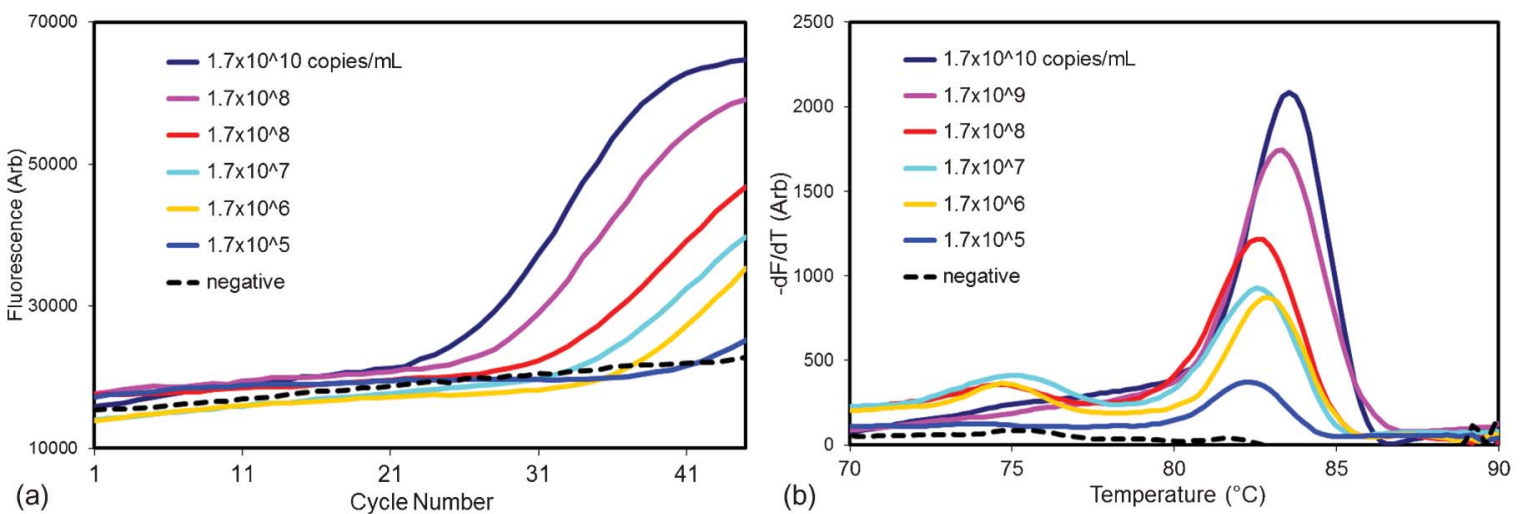

Fig. 3 Using gel strip PCR to detect blood-borne BKV. BKV DNA was spiked into $1: 10$ diluted blood (a) real-time PCR data and (b) MCA curves. BKV DNA used to spike whole blood was precisely titered using Taqman PCR prior to use in characterizing the gel strip method. These results were verified in five independent experiments.

previously. ${ }^{9}$ The PCR recipe is provided in the supplementary materials. $\dagger^{\dagger}$

The gel was polymerized for $30 \mathrm{~min}$ under $370 \mathrm{~nm}$ UV light and then the cassette was desiccated overnight under 24 inch $\mathrm{Hg}$ in a vacuum oven (Isotemp Vacuum Oven 281A, Fisher Scientific). In gel capillaries, ${ }^{6}$ the position of the channel created by desiccation is random. However because gel strips are fixed in their position by the glass cover slip, when desiccation creates a space for the blood to be delivered by capillary force, the channel is always underneath the gel as shown in the Fig. 1(d). For sample delivery, each gel strip received $8.5 \mu \mathrm{L}$ of $10 \times$ diluted blood spiked with BKV DNA. Hydration of the gel with blood takes about 10 min. A cartoon of the entire cassette containing 12 gel strips is seen in Fig. 2(a) and a real gel strip cassette is shown in Fig. 2(b). Fig. 2(c) shows photographs of (i) desiccated gel strips and (ii) gel strips hydrated with blood, and (iii) a CCD image of fluorescent signal and (iv) a photograph of strips after the PCR is completed.

The prototype instrument used for PCR and MCA was described earlier. ${ }^{6}$ PCR was performed with a pre-denaturation step of 6 min at $94{ }^{\circ} \mathrm{C}, 45$ cycles with $94^{\circ} \mathrm{C}$ for $15 \mathrm{~s}, 60^{\circ} \mathrm{C}$ for $20 \mathrm{~s}$, and $72{ }^{\circ} \mathrm{C}$ for $20 \mathrm{~s}$, followed by a final extension step of $120 \mathrm{~s}$ at 72 ${ }^{\circ} \mathrm{C}$. During the PCR, CCD images were taken at $5 \mathrm{~s}$ into each extension step. MCA to validate the PCR product was performed from $65-90{ }^{\circ} \mathrm{C}$ and $\mathrm{CCD}$ images were taken at every $0.2{ }^{\circ} \mathrm{C}$. Analysis of the CCD images to obtain the PCR and MCA data was described previously. ${ }^{1}$

The real-time PCR curves obtained with $1: 10$ blood containing different amounts of BKV DNA are shown in Fig. 3(a) while the MCA peaks are shown in Fig. 3(b). These copy numbers vary from $1.43 \times 10^{7}$ to 143 copies per gel strip. These results were replicated with blood from multiple individuals 5 times. The melt temperatures can vary by about $1.2^{\circ} \mathrm{C}$. These shifts were observed with conventional methods as well. ${ }^{8}$

In addition to $1: 10$ diluted blood we also tested the $1: 5$ diluted blood and undiluted blood to investigate the influence of the amount of blood on the PCR. The MCA data with different concentrations of blood with $1.7 \times 10^{9}$ copies per $\mathrm{mL}$ of BKV are shown in Fig. 4. We found that a $1: 5$ dilution of blood gives a weaker peak than $1: 10$ diluted blood. No product was amplified from undiluted blood. Dilutions of blood lower than $1: 10$ most probably inhibit the PCR.

To confirm the ability of the gel strip method to detect BKV in clinical samples, we tested two raw blood samples from transplant patients and could detect BKV viremia of $\sim 5 \times 10^{4} \mathrm{BKV} \mathrm{ml}^{-1}$. We also successfully detected the FGFR2 gene in human genomic DNA from unprocessed blood samples.

\section{Conclusion}

Here we communicate a strategy to test for pathogens in whole blood without the lengthy purification and DNA extraction steps that are usually performed by highly trained staff in a central laboratory environment. Blood is delivered under the gel strip reaction unit into a wax trench that forms part of a PCR cassette. The channel for sample delivery is formed when the desiccated gel containing all reagents except template shrinks and pulls away from the bottom of the cassette. This innovative but simple

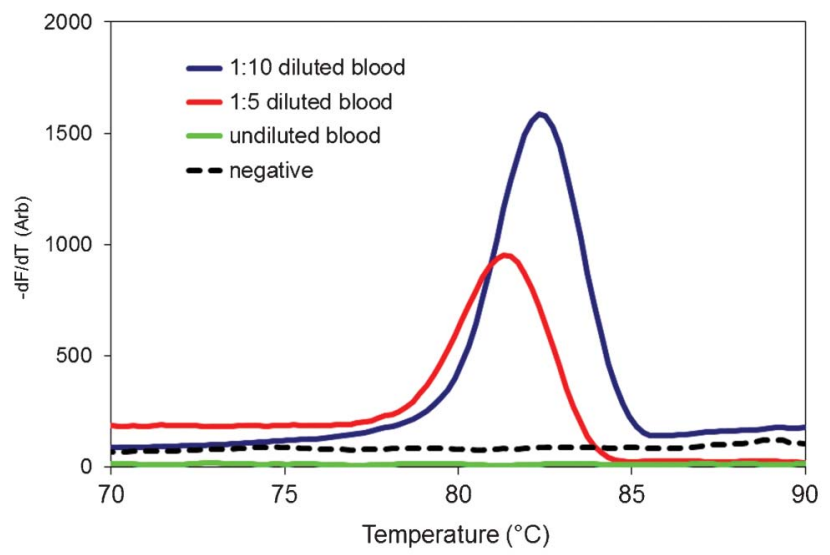

Fig. 4 Testing of blood dilutions. MCA curves are shown for testing a range of blood dilutions, all spiked with the same amount of BKV DNA. These results were verified in two independent experiments. 
sample delivery method leaves opaque blood components underneath the gel strip, thereby avoiding problematic masking of the PCR fluorescent signal by the dark color and solid components of whole blood. This permits accurate monitoring from the topside of the gel strip of the binding by intercalating dye to the amplified DNA. Testing of unprocessed whole blood in a miniaturized device will facilitate immediate near-point of care testing for infectious agents in emergency wards and clinics leading to improved treatment and control of spread within the community.

Funded by an interdisciplinary team grant from Alberta Innovates Health Solutions.

\section{References}

1 D. P. Manage, J. Lauzon, A. Atrazhev, R. Chaveli, R. A. Samuel, B. Chan, Y. C. Morrissey, W. Gordy, A. L. Edwards, K. Larison, S.
Y. Yanow, J. P. Acker, G. Zahariadis and L. M. Pilarski, Lab Chip, 2013, 13, 2576-2584.

2 O. T. Campbell-Yesufu and R. T. Gandhi, Clin. Infect. Dis., 2011, 52, 780-787.

3 L. D. Kramer, J. Li and P. Y. Shi, Lancet Neurol., 2007, 6, 171-181.

4 R. Tuteja, FEBS J., 2007, 274, 4670-4679.

5 T. Wilkins, D. Zimmerman and R. R. Schade, Am. Fam. Physician, 2010, 81, 965-972.

6 M. D. Reploeg, G. A. Storch and D. B. Clifford, Clin. Infect. Dis., 2001, 33, 191-202.

7 D. P. Manage, Y. C. Morrissey, A. J. Stickel, J. Lauzon, A. Atrazhev, J. P. Acker and L. M. Pilarski, Microfluid. Nanofluid., 2011, 10, 697-702.

8 B. J. Taylor, K. A. Martin, E. Arango, O. M. Agudelo, A. Maestre and S. K. Yanow, Malar. J., 2011, 10, DOI: 10.1186/1475-2875-10244.

9 A. Atrazhev, D. P. Manage, A. J. Stickel, H. J. Crabtree, L. M. Pilarski and J. P. Acker, Anal. Chem., 2010, 82, 8079-8087. 